\title{
Time taken for a primary tumor to metastasize to the brain and the overall survival of patients with brain metastasis: An analysis of outcomes and factors affecting survival
}

\author{
Peiyu Hao ${ }^{\mathrm{a}, 1}$, Limei Chen ${ }^{\mathrm{a}, 1}$, Yuxi Ge ${ }^{\mathrm{b}}$, Yaqian You $^{\mathrm{a}}$, Gen Yan ${ }^{\mathrm{b}, \mathrm{c}, *}$ and Zhe-Wu Jin ${ }^{\mathrm{d}, *}$ \\ ${ }^{a}$ Department of Public Health and Preventive Medicine, Wuxi School of Medicine, Jiangnan University, \\ Wuxi, Jiangsu, China \\ ${ }^{\mathrm{b}}$ Department of Radiology, Affiliated Hospital of Jiangnan University, Wuxi, Jiangsu, China \\ ${ }^{\mathrm{c}}$ Deparment of Radiology, The Second Affiliated Hospital of Xiamen Medical College, Xiamen, Fujian, \\ China \\ ${ }^{\mathrm{d}}$ Department of Anatomy, Wuxi School of Medicine, Jiangnan University, Wuxi, Jiangsu, China
}

\begin{abstract}
.
BACKGROUND: Brain metastasis (BM) is one of the main causes of high morbidity and mortality in cancer patients.

OBJECTIVE: To evaluate the factors that influenced the survival time of patients with primary cancer and survival time after BM.

METHODS: Ninety patients with BM diagnosed by magnetic resonance imaging (MRI) were included in the study. We retrospectively analyzed the time to brain metastasis (TTB), overall survival time (OS1) and survival time after BM (OS2). The Kaplan-Meier method and Cox regression analysis were used for survival analyses.

RESULTS: The median TTB was 12.0 (95\% CI: 9.2-14.8 months), the median OS1 was 31.0 (95\% CI: 25.8-35.2 months) and the median OS2 was 14.0 (95\% CI: 10.9-17.1 months). Surgical excision of the primary tumor was an independent factor for a prolonged TTB $(p<0.000)$ and prolonged OS1 $(p<0.000)$. A single intracranial metastatic lesion was an independent protective factor for prolonged OS1 $(p=0.011)$ and prolonged OS2 $(p=0.050)$. TTB, OS1 and OS2 were analyzed with Gender $(p<0.000,<0.000$, and $=0.017$, respectively).

CONCLUSIONS: It suggests that TTB can be prolonged by primary tumor resection. Furthermore, women with a prolonged TTB and single intracranial metastasis are associated with high OS. These were helpful for the clinical treatment of BM patients before brain metastasis.
\end{abstract}

Keywords: Primary tumor, brain metastasis, time to brain metastasis, overall survival, retrospective analysis, surgery

\footnotetext{
${ }^{1}$ These authors contributed equally to this work.

${ }^{*}$ Corresponding authors: Gen Yan, Department of Radiology, The Second Affiliated Hospital of Xiamen Medical College, 556 Shengguang Road, Xiamen, Fujian 361021, China. E-mail: gyan@ stu.edu.cn. Zhe-Wu Jin, Department of Anatomy, Wuxi School of Medicine, Jiangnan University, No. 1800 Lihu Avenue, Wuxi, Jiangsu 214122, China. Tel.: +86 510 8519 7079; Fax: +86 5108519 3570; E-mail: zwjin@jiangnan.edu.cn.
} 


\section{Introduction}

Brain metastasis $(\mathrm{BM})$ is the most common intracranial malignant tumor in adults. BM usually metastasizes from tumor cells outside of the brain tissue. The incidence of BM is certainly much higher than that of primary intracranial tumors, and intracranial metastases found in 8.5-9.6\% of patients with malignant tumors [1,2]. The most common metastatic organ include lung (50\%), breast (15\%-20\%), melanoma (5\%-10\%), kidney (7\%), and colon (4\%-6\%). In BM from lung cancer, non-small cell lung cancer is the most common primary type, accounting for approximately 35-45\% [3]. A small number of major lesions cannot be detected, and BM can be single or multiple. However, magnetic resonance imaging (MRI) revealed that multiple lesions are more commonly observed [3,4]. BM found in 20\% of cancer patients, with the improvement of imaging technology, BM patients can be diagnosed as early as possible and then effective systemic treatments can be adopted, the true incidence rate should be higher [5-7].

Currently, the main treatment measures for BM include surgery, whole-brain radiotherapy (WBRT), stereotactic radiotherapy (RT), and chemotherapy [4]. However, the treatment and prognosis of BM are still controversial. To determine the developmental pattern of BM, many clinical researchers have evaluated many prognostic factors as well as the use of clinical interventions (such as RT and chemotherapy) to analyze whether there were significant differences considering the impact of different factors on the survival rate of patients with BM. Studies have shown differences in survival rates between men and women as well as considering the number of BM [8,9]. Different clinical interventions, such as resection of metastatic lesions, also affect prognosis [10]. In addition, the survival rate of patients with lung adenosquamous carcinoma with $\mathrm{BM}$ is also significant due to the different time from primary tumor to brain metastasis (TTB) [11]. However, in these studies, the diagnosis of BM was the clinical threshold and the characteristics of tumor progression after BM were analyzed, ignoring the effect of tumor progression on the survival rate during the TTB. In recent studies, usually based on the diagnosis of BM, collected and analyzed of patients clinical characteristics and treatment. Analyze the survival of BM patients after brain metastasis, find out the prognostic factors that have statistical significance for survival time. However, these studies did not include the clinical features before BM, and the impact of primary tumor progression on TTB. Effective intervention before the primary tumor metastasizes to the brain will help to better delay the process of brain metastasis and improve the quality of life. Therefore, in the current study, BM was the research boundary point on the basis of the original study; we then analyzed the characteristics of the different types of primary cancers before and after metastasis and finally added up the two time periods for comprehensive analysis. Moreover, analysis of clinical data of BM patients after brain metastasis combined the two periods for comprehensive analysis.

We analyzed the characteristics of primary cancer in 90 patients with BM and evaluated the factors that influenced the survival time of patients with primary cancer and survival time after BM. We defined the time from the date of diagnosis of the primary tumor to the diagnosis of BM as the TTB, the time from the diagnosis of the primary tumor to the end of the study as the overall survival time (OS1), and the time from the diagnosis of BM to the end of the study as the survival time after BM (OS2). Owing to the limitations of resection of $\mathrm{BM}[12,13]$, we hypothesized that resection of the primary tumor can delay the occurrence of BM and increase the total survival time of patients during the TTB.

The results of our study will provide the basis for clinical treatment and improve the existing treatment model.

1. We collected clinical data of BM patients before brain metastasis, analyzed their TTB survival status, overcame the previous research that focused survival situation after brain metastasis, and provide a reference for the early treatment of patients with brain metastasis. 
2. Moreover, we collected data of BM patients after brain metastasis, analyzed the survival of BM patients after brain metastasis. We combined two periods and comprehensively analyzed the impact of various factors on survival time, the last three stages of survival. The results reflecting the different prognostic factors that affect survival time of BM patients.

We summarized the demographic and clinical characteristics of 90 patients with BM. We analyzed the factors based on TTB, OS1, and OS2.

\section{Materials and methods}

Written informed consent was obtained from each participant, and the study protocol was approved by the Medical Ethics Committee of the Affiliated Hospital. All patient-related information was confidential. The criteria for inclusion in this retrospective study included a diagnosis of a primary tumor, BM, and RT evaluation.

The current retrospective study was performed in Wuxi City, Jiangsu Province, China. We selected the patients first. Eligible patients were those living in this city for at least 3 years and who were planning to be admitted to the Affiliated Hospital of Jiangnan University for treatment. The study was conducted from May 2010 to May 2018. The inclusion criteria were BM patients (diagnosed by MRI enhancement) with initial metastasis that met the surgical indications, and the patients lost to follow-up and transferred to hospital were excluded. Next, we determined the required research variables, then arranged the patient's medical records, continued following up the patients or their families. We collected the necessary records of patient including clinical trial data in time to ensure the accuracy and consistency of the data collection and entry process. Simultaneously, the data of all the patients were strictly verified, and the diagnostic criteria for each case were checked if they were unified, if the necessary examination items were complete, if the observation indicators were complete, and if the efficacy criteria were consistent. These patients were histologically diagnosed with different primary tumors, and the occurrence of BM was checked using enhanced MRI of the brain.

Survival analysis was used mainly in the study. We used the Kaplan-Meier test to analyze the single variable, and the factors with $p$-values less than 0.05 were selected. Log-rank test was used to compare survival curves. The factors with statistically significance in univariate analysis were summarized and included in Cox regression model for multivariate analysis to further determine the independent prognostic factors related to OS.

The main endpoints of the study were TTB, OS1, and OS2. OS1 was calculated from the time of diagnosis of the primary tumor to the end of the study. OS2 was defined as the time from BM to the end of the study. Other relevant information were obtained by interview and confirmed by medical records, such as gender, age, primary tumor type, primary tumor control, extracranial tumor control, BM occurrence time, BM quantity, BM location, and BM treatment information (whether the primary tumor was treated with surgery and whether the primary tumor was treated with RT or chemotherapy after BM).

Survival data are expressed in months. The 1-year, 2-year, and 3-year survival rates were calculated. All descriptive survival data were presented with the $95 \%$ confidence interval $(95 \% C I)$. We generated a stacked bar chart showing the relationships between the survival data for all cases. All the reported $C I \mathrm{~s}$ were bilateral, and p-values less than 0.05 were considered statistically significant. All statistical analysis and graphics generation were performed using SPSS ${ }^{\circledR}$ software version 21.0 (SPSS Inc., Chicago, IL, USA). 

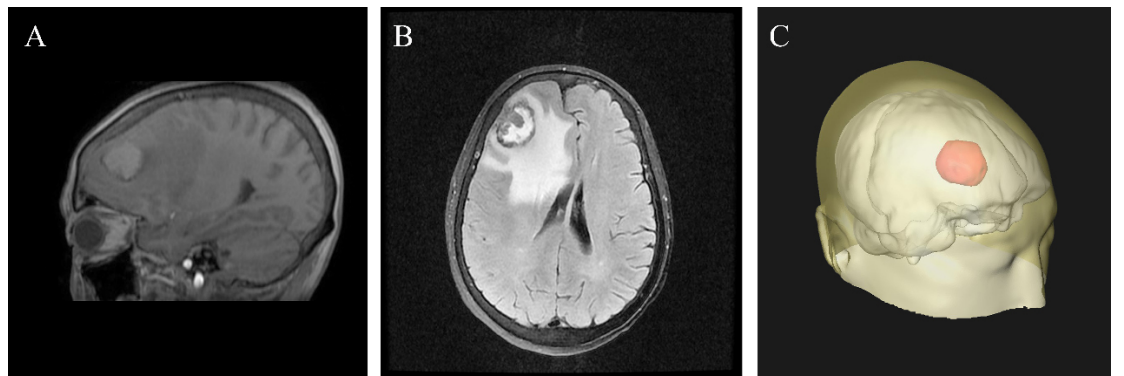

Fig. 1. Imaging characteristics of metastatic brain lesions. (A) On sagittal GRE T1 map, the tumor is located in the medullary junction of the right frontal lobe with irregular contour. The sign of the tumor is slightly lower in the periphery and slightly higher in the middle. (B) T2 flair of transverse lesion showed that the tumor presented mixed signals with low signal in the peripheral area and slightly high signal in the middle area. The edge of the tumor is rough with a large edema surrounding it and the midline of the prefrontal structure is left-skewed. (C) The three-dimensional fluoroscopy clearly shows the overall structure of the brain and the location of the tumor, providing an intuitive and comprehensive imaging basis for preoperative positioning.
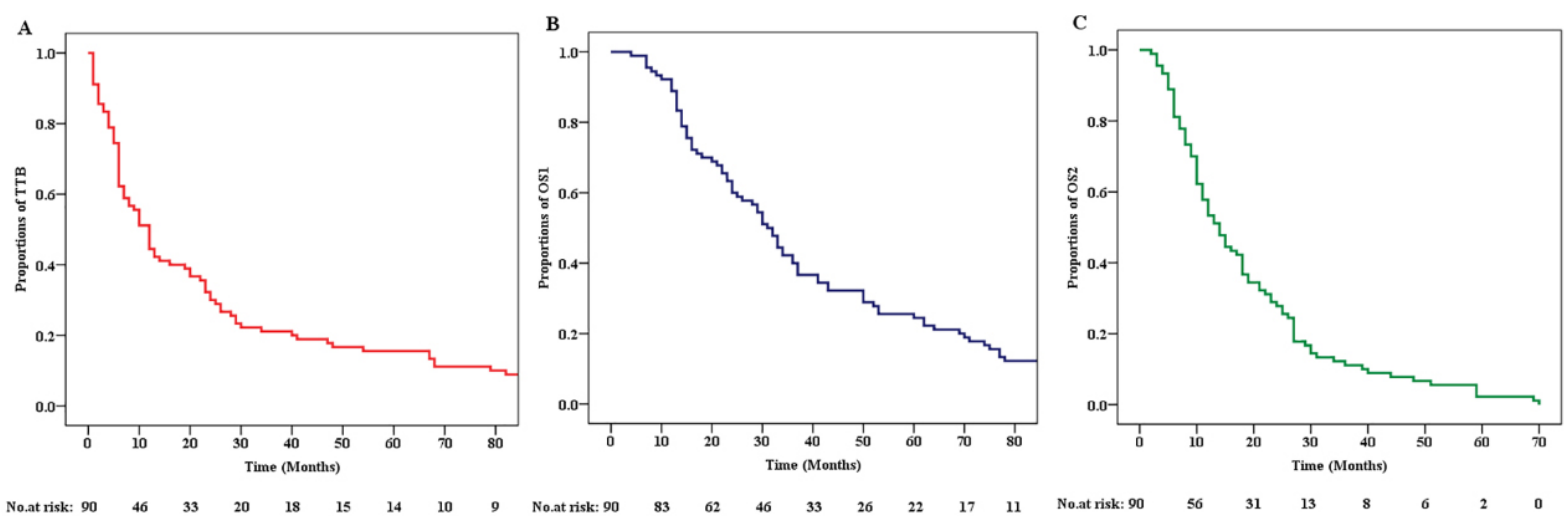

Fig. 2. Kaplan-Meier curves showing the overall survival of patients with primary brain metastasis. (A) Kaplan-Meier curve showing the TTB of patients with primary tumor brain metastasis. (B) Kaplan-Meier curve showing the OS1 of patients with primary tumor brain metastasis. (C) Kaplan-Meier curve showing the OS2 of patients with primary tumor brain metastasis. TTB: time of brain metastasis, OS1: overall survival 1, time of diagnosis of the primary tumor to the end of the study. OS2: overall survival 2, time from BM to the end of the study.

\section{Results}

\subsection{Demographic and clinical characteristics of patients}

The demographic and clinical characteristics of the 90 patients included in the random sample are shown in Table 1. The primary tumor in these patients was mostly lung cancer (70.0\%), and adenocarcinoma was the most common tumor type (60.0\%). BM lesions were more common in the supratentorial part of the brain $(58.9 \%)$ or in the whole brain $(32.2 \%)$. Multiple BM lesions $(65.6 \%)$ were more common than single metastases (34.4\%) when BM was first found. In most patients, BM lesions were accompanied by edema $(72.2 \%)$ but without any hemorrhage $(93.3 \%)$ or necrosis $(93.3 \%)$. Figure 1 shows the imaging characteristics and three-dimensional fluoroscopy of metastatic brain lesions. The median TTB was 12.0 months (95\% CI: 9.2-14.7, Fig. 2A). The median OS1 was 31.0 months (95\% CI: 25.8-35.2, Fig. 2B). The median OS2 was 14.0 months (95\% CI: 10.9-17.1, Fig. 2C). 
Table 1

Patient demographic and clinical characteristics $(n=90)$

\begin{tabular}{|c|c|}
\hline Characteristic & No. $(\%)$ \\
\hline \multicolumn{2}{|c|}{ Median survival time (months) } \\
\hline TTB & 12.0 \\
\hline OS1 & 31.0 \\
\hline OS2 & 14.0 \\
\hline \multicolumn{2}{|l|}{ Gender } \\
\hline Male & $48(53.3)$ \\
\hline Female & $42(46.7)$ \\
\hline \multicolumn{2}{|l|}{ Age (year) } \\
\hline$<60$ & $42(46.7)$ \\
\hline$\geqslant 60$ & $48(53.3)$ \\
\hline \multicolumn{2}{|l|}{ Category 1} \\
\hline Lung cancer & $63(70.0)$ \\
\hline Breast cancer & $15(16.7)$ \\
\hline Colon cancer & $8(8.9)$ \\
\hline Other cancer & $4(4.4)$ \\
\hline \multicolumn{2}{|l|}{ Category 2} \\
\hline Adenocarcinoma & $54(60.0)$ \\
\hline Non-small lung cancer & $7(7.8)$ \\
\hline Small cell lung cancer & $10(11.1)$ \\
\hline Squamous carcinoma & $8(8.9)$ \\
\hline Ductal carcinoma & $7(7.8)$ \\
\hline Other & $4(4.4)$ \\
\hline \multicolumn{2}{|l|}{ Primary tumor control } \\
\hline Yes & $71(78.9)$ \\
\hline No & $19(21.1)$ \\
\hline \multicolumn{2}{|l|}{ Extracranial tumor control } \\
\hline Yes & $51(56.7)$ \\
\hline No & $39(43.3)$ \\
\hline \multicolumn{2}{|l|}{ No. of brain metastasis } \\
\hline 1 & $31(34.4)$ \\
\hline$\geqslant 2$ & $59(65.6)$ \\
\hline \multicolumn{2}{|l|}{ Location } \\
\hline Supratentorial & $53(58.9)$ \\
\hline Subtentorial & $8(8.9)$ \\
\hline Both & $29(32.2)$ \\
\hline \multicolumn{2}{|l|}{ Border } \\
\hline Well-defined & $64(71.1)$ \\
\hline Ill-defined & $26(28.9)$ \\
\hline \multicolumn{2}{|l|}{ Morphology } \\
\hline Regular & $65(72.2)$ \\
\hline Irregular & $25(27.8)$ \\
\hline \multicolumn{2}{|l|}{ Edema } \\
\hline Yes & $65(72.2)$ \\
\hline No & $25(27.8)$ \\
\hline \multicolumn{2}{|l|}{ Necrosis } \\
\hline Yes & $6(6.7)$ \\
\hline No & $84(93.3)$ \\
\hline \multicolumn{2}{|l|}{ Hemorrhage } \\
\hline Yes & $6(6.7)$ \\
\hline No & $84(93.3)$ \\
\hline
\end{tabular}

TTB: time to brain metastasis; OS: overall survival. OS1 in months: From the primary tumor diagnosis to the end of the follow-up; OS2 in months: From the date of brain metastasis diagnosis. 

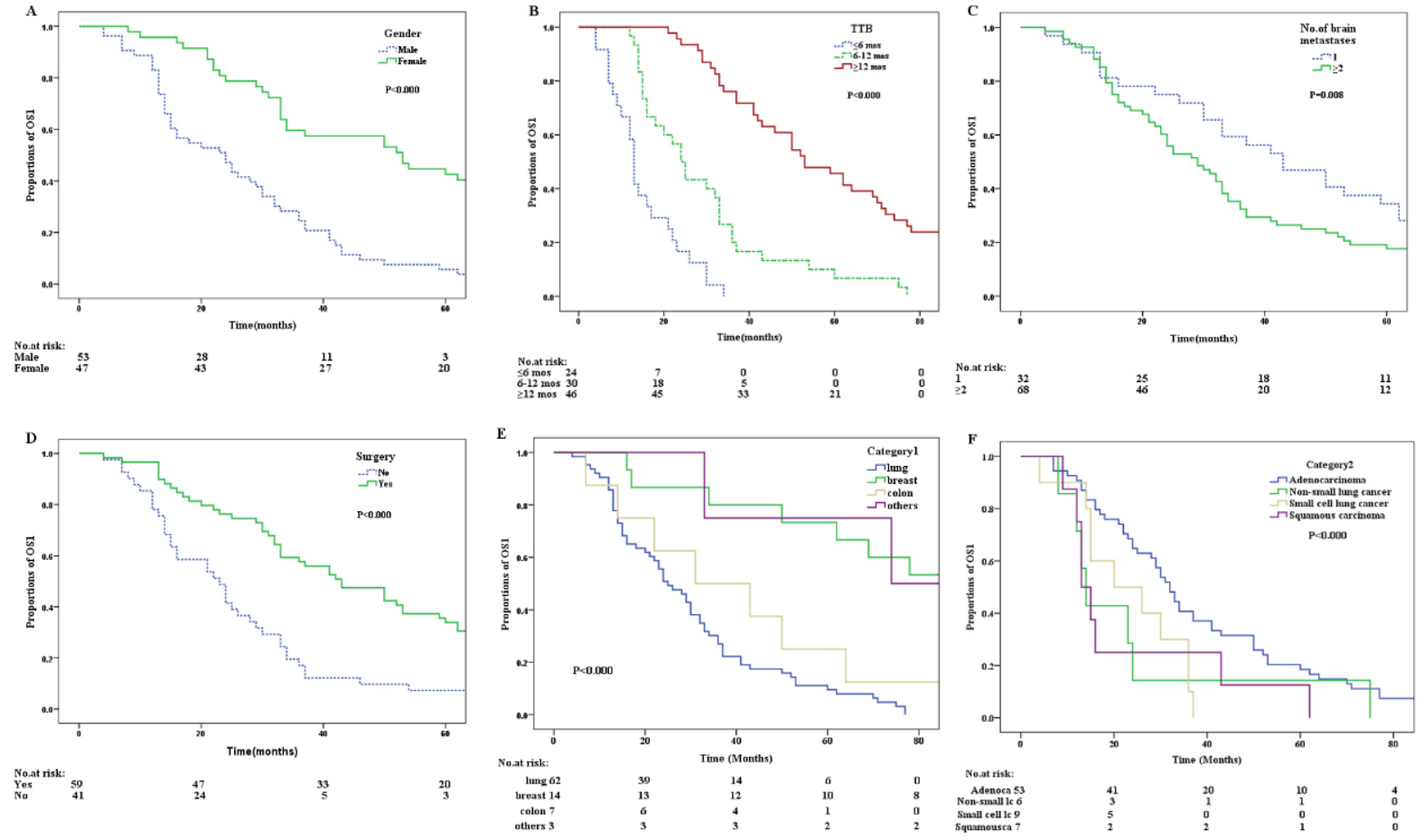

Fig. 3. Kaplan-Meier curves of factors that independently influenced OS1. (A) Kaplan-Meier curve for OS1 according to Gender. (B) Kaplan-Meier curve for OS1 of patients according to the TTB. (C) Kaplan-Meier curve for OS1 of patients according to the number of brain metastasis. (D) Kaplan-Meier curve for OS1 of patients according to whether they underwent surgery. (E) Kaplan-Meier curve for OS1 of patients according to the difference in the location of the primary tumor. (F) Kaplan-Meier curve for OS1 of patients according to different pathological types of primary tumors.

\subsection{Single factor and multiple factor survival analysis of OS data}

The results of univariate survival analysis by using the Kaplan-Meier method and multivariate survival analysis by using Cox regression analysis for TTB are shown in Table 2. The results showed that female (Gender), < 60 (Age), breast cancer (category 1), ductal carcinoma (category 2), single BM, and undergoing surgery were predictors of a prolonged TTB. The TTB was as follows considering the following significant predictors: gender (male: 7.0 vs. female: 26.0$)$, age ( $<60: 13.0$ vs. $\geqslant 60: 9.0$ ), category 1 (lung cancer: 7.0 vs. breast cancer: 68.0 vs. colon cancer: 25.0 vs. other cancer: 47.0 ), category 2 (adenocarcinoma: 12.0 vs. non-small lung cancer: 4.0 vs. small cell lung cancer: 6.0 vs. squamous carcinoma: 3.0 vs. ductal carcinoma: 107.0 vs. others: 47.0$)$, number of BM (1: 22.0 vs. $\geqslant 2: 10.0)$ and surgery (yes: 23.0 vs. no: 6.0 ). However, multivariate analysis revealed that only female (Gender) and undergoing surgery were the independent predictors of a prolonged TTB ( $p=0.040$ and 0.001 , respectively).

The results of univariate survival analysis by using the Kaplan-Meier method and multivariate survival analysis by using Cox regression analysis for OS1 are shown in Table 3. The results showed that female (Gender), breast cancer (category 1), ductal carcinoma (category 2), TTB $>6$ months, single BM, and undergoing surgery were predictors of a prolonged OS1. The OS1 was as follows considering the following significant predictors: Gender (male: 20.0 vs. female: 53.0, $p<0.000$, Fig. 3A), category 1 (lung cancer: 25.0 vs. breast cancer: 86.0 vs. colon cancer: 31.0 vs. other cancer: 74.0 ), category 2 

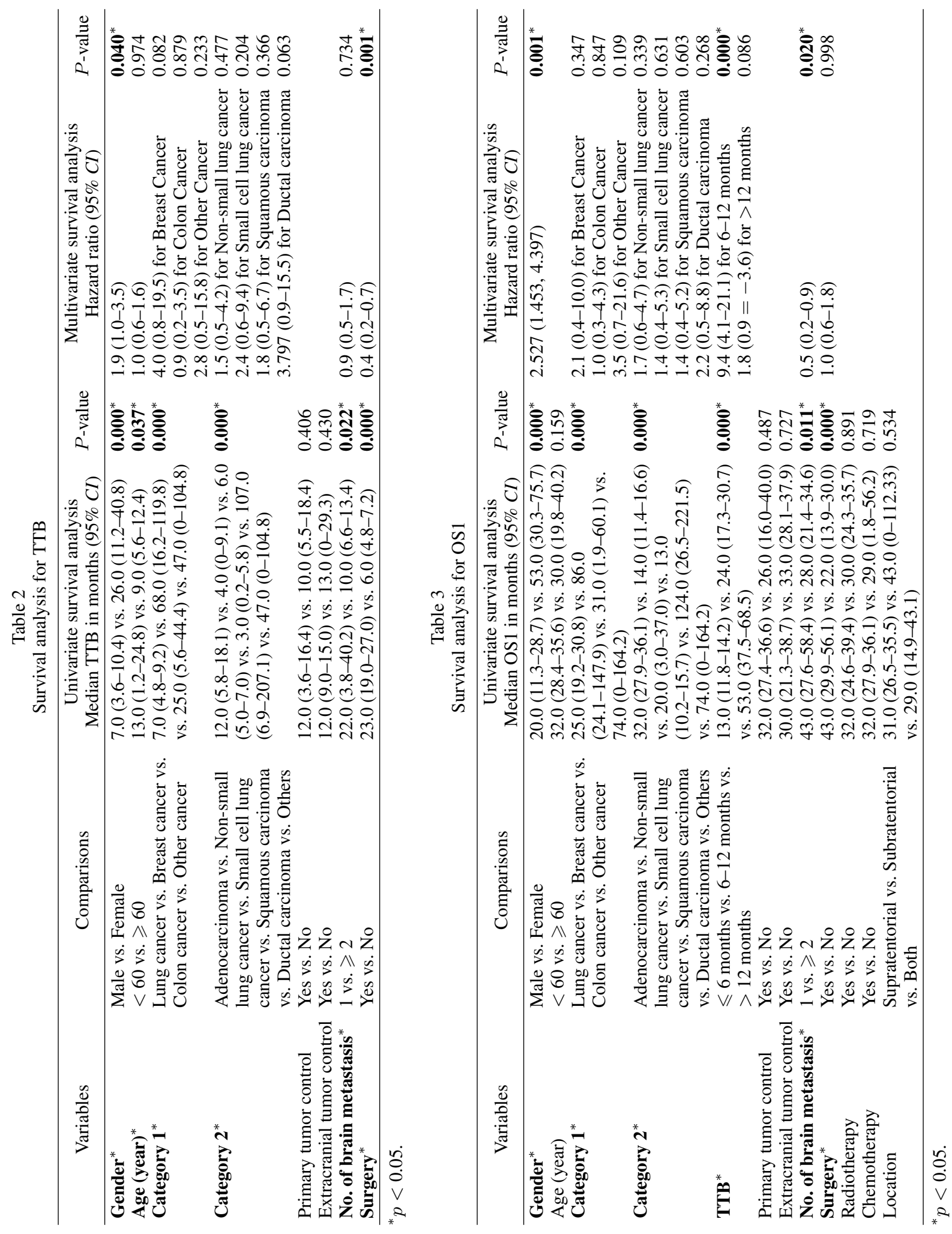


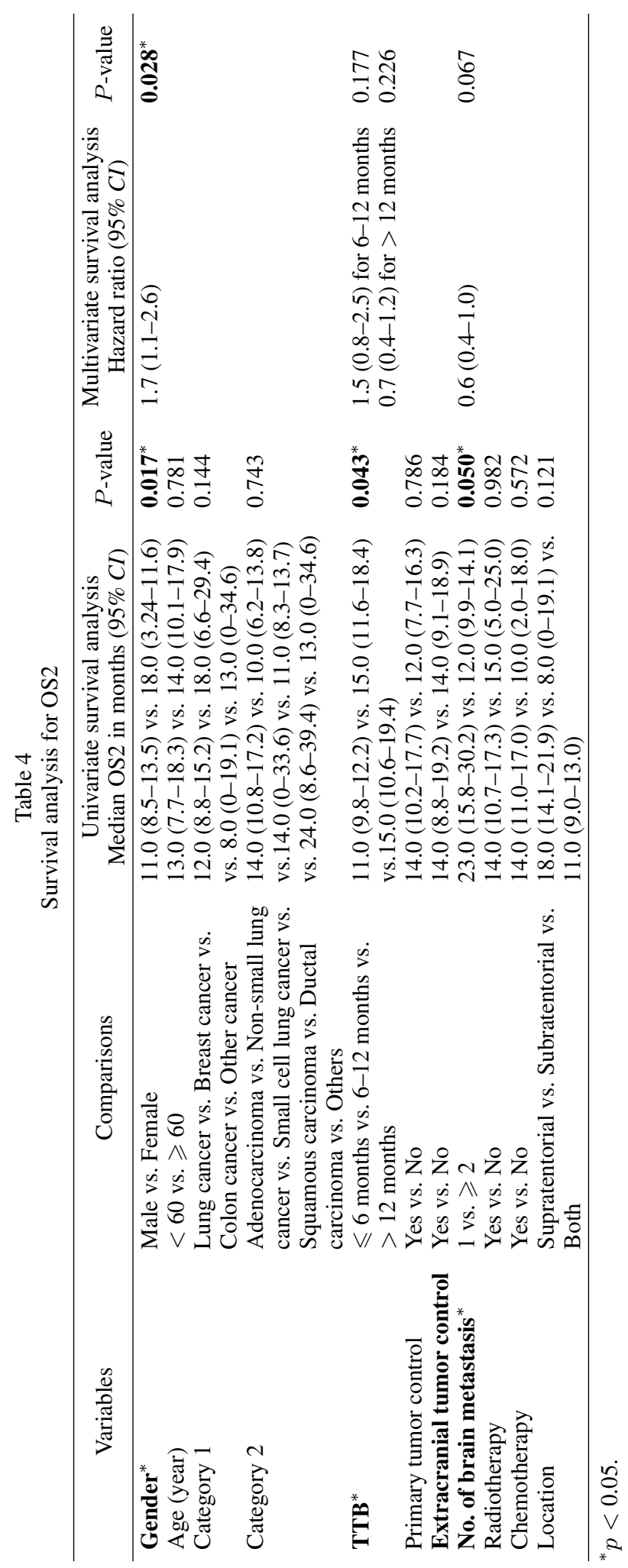



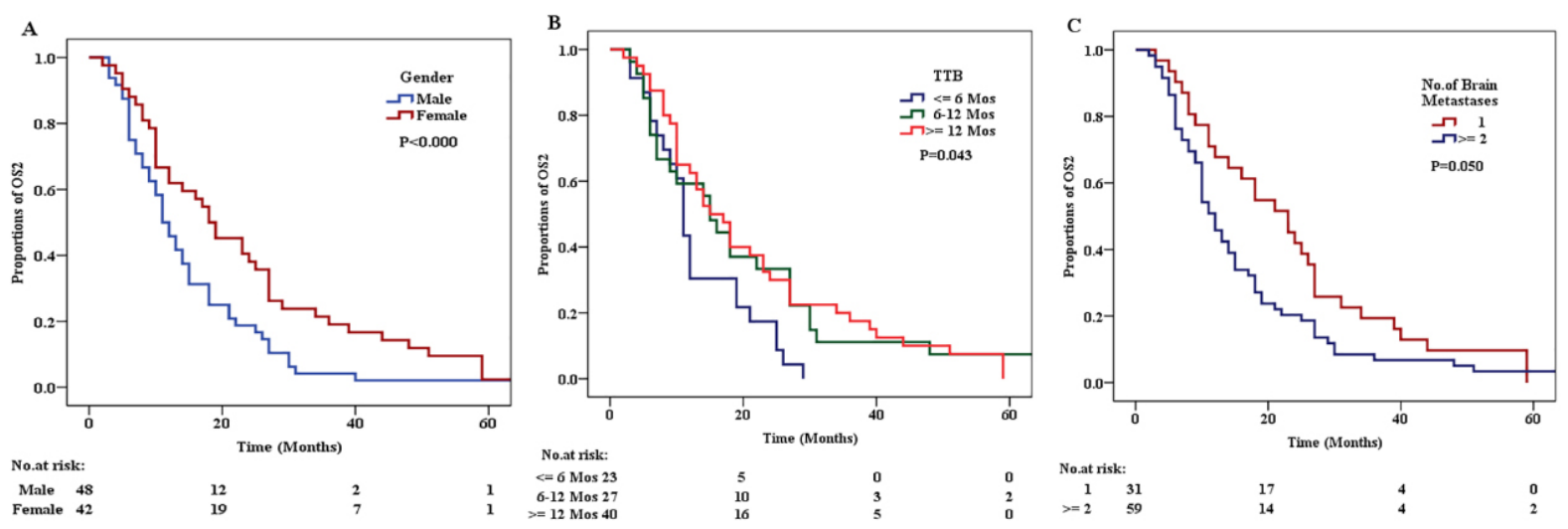

Fig. 4. Kaplan-Meier curves of factors that independently influenced OS2. (A) Kaplan-Meier curve for OS2 according to Gender. (B) Kaplan-Meier curve for OS2 according to the TTB. (C) Kaplan-Meier curve for OS2 of patients according to the number of BM lesions.

(adenocarcinoma: 32.0 vs. non-small lung cancer: 14.0 vs. small cell lung cancer: 20.0 vs. Squamous carcinoma:13.0 vs. Ductal carcinoma: 124.0 vs. Others: 4.0$)$, TTB ( $\leqslant 6$ months 13.0 vs. $6-12$ months: 24.0 vs. $>12$ months: $53.0, p<0.000$, Fig. 3 B), number of BM (1: 43.0 vs. $\geqslant 2: 28.0, p=0.011$, Fig. 3C), and surgery (yes: 43.0 vs. no: $22.0, p<0.000$, Fig. 3D). However, multivariate analysis revealed that female (Gender), single BM, and TTB of 6-12 months were independent predictors of a prolonged OS1 ( $p=0.001,=0.020$, and $<0.000$, respectively; Table 3 ).

The results of univariate survival analysis by using the Kaplan-Meier method and multivariate survival analysis by using Cox regression analysis for OS2 are shown in Table 4. The results showed that female (Gender), TTB $>6$ months, and single BM were predictors of a prolonged OS2. The OS2 was as follows considering the following significant predictors: Gender (male: 11.0 vs. female: 18.0, $p<0.000$, Fig. 4A), TTB ( $\leqslant 6$ months: 11.0 vs. $6-12$ months: 15.0 vs. $>12$ months: $15.0, p=0.043$, Fig. 4 B), and number of BM (1: 23.0 vs. $\geqslant 2: 12.0, p=0.050$, Fig. 4C). However, multivariate analysis revealed that only female (Gender) ( $p=0.028$, Table 4$)$ was an independent predictor of a prolonged OS2.

\section{Discussion}

In the current study, we analyzed the survival data of 90 patients with primary malignant tumors. Univariate analysis revealed that the median survival time of all the patients (including those with BM at the time of diagnosis) was approximately 31 months. Gender, age, category 1, category 2, number of BM, and surgery were independent predictors of TTB. Gender, category 1, category 2, TTB, number of BM, and surgery were independent predictors of OS1. Gender, TTB, and number of BM were independent predictors of OS2.

Currently, survival analysis of patients with BM treated with resection is mainly focused on the removal of BM, while no relevant study has evaluated whether the primary tumor should be removed via resection. In fact, many studies have focused on patient survival after excision of BM; however, the results of these studies were controversial regarding whether surgical treatment could benefit patients with primary tumors with BM. Surgical resection plays a key role in a single brain metastasis. Surgical resection can remove large, life-threatening tumors, and is also indispensable for providing or confirming pathological diagnosis. Some studies have shown that resection of BM can improve survival [14-16]. However, the 
other study has pointed out that the cause of death in patients with brain metastases is that the primary disease is aggravated by $50 \%$, and neurological death due to brain metastases or pia maters accounts for $30 \%$ [17].

The role of surgery in patients with multiple brain metastases is unclear. In the treatment of multiple brain metastases, WBRT and chemotherapy are still the main treatments. The prognosis of this condition is poor, and patients with four or more brain tumors are usually not treated with surgery. The technical difficulty of the operation is also high, and the surgical decision must be made according to the patient's clinical situation. Studies have pointed out that the impact of primary tumor control on survival time is more important than the number of brain lesions. However, compared with patients whose primary tumor is controlled, uncontrolled patients are more likely to have more brain metastases [18]. A few studies have evaluated the effect of surgical resection in patients with two or more brain metastases and have reached conflicting results. A prospective observational study by Yamamoto et al. found that in the absence of WBRT, the survival rate of 5-10 patients with brain metastases treated with stereotactic radiosurgery was lower than that of patients with only 2-4 brain metastases [19]. There was no statistical difference ( $p=$ 0.890). In the current study, we found that resection of the primary tumor was not significantly associated with the total survival time of patients with BM. To the best of our knowledge, the current study is the first to show that removal of the primary tumor can prolong TTB although it may cause the primary tumor to metastasize to the brain later. Although there was no significant association between removal of the primary tumor and prolonged survival, previous studies have shown poor quality of life in patients with BM. Therefore, we believe that resection can improve the local control of primary tumors that are easy to metastasize to the brain and improve the quality of life when they are removed in the early stage of metastasis.

A few epidemiological studies have shown that different RT regimens can prolong survival in patients with BM. For example, a survey published in 2019 revealed that, compared with WBRT alone, WBRT + enhanced RT (RTB) significantly improved intracranial progression-free survival time of patients with BM of non-small cell lung cancer [20]. Rades et al. found similar results that adding RT to WBRT improved intracerebral control in patients with BM [21]. In addition to the effect after WBRT, intracerebral control can be significantly improved via stereospecific enhancement alone. OS improved only in patients with a single BM, but not in the entire cohort [18]. We did not find any significant relationship between RT and chemotherapy and the survival time (TTB, OS1, and OS2). Some of the observed inconsistencies might be owing to the differences in the study population and the sample size, although we are unable to confirm the reason for the difference between the results of previous studies and our results. Nevertheless, the adverse effects of RT cannot be ignored. In fact, many studies have described the adverse effects of $\mathrm{RT}$, such as radionecrosis [22,23], which is difficult to diagnose and treat.

Multivariate analysis revealed that Gender and surgery were important factors that influenced TTB, and that resection of the primary tumor was a predictor of prolonged TTB. Removal of the primary tumor before BM can delay metastasis and prolong TTB. However, whether the primary tumor lesion was removed did not significantly influence OS1. On multivariate survival analysis, we found that a prolonged TTB was a prolonged independent predictor of OS1. Gender, TTB, and number of BM were important prognostic factors for OS1. Moreover, patients with lung adenosquamous carcinoma with longer TTB have a higher survival rate [11]. It was similar with our results. In addition, in our study, Gender and number of BM were important prognostic factors for OS2. Therefore, it is recommended that the primary tumor be removed when surgery is feasible. However, because the tumors spread throughout the body, removal of the tumor lesion was not possible in many patients. For such patients, 4-6 cycles of standard chemotherapy plus RT are required. The number of BM plays an important role in the prognosis of 
patients. The survival rate of patients with single BM is higher than that of patients with multiple BM, it was similar to the previous study by Eigentler et al. [24]. It associated with the progression of tumor metastasis, where multiple metastasis can compress the brain tissue and cause more damage, accelerating the progression of the disease.

The OS data were shown according to two different starting times. In clinical experience, the performance of patients with BM deteriorates, and the survival time is very limited. This study is the first to investigate factors that may influence the survival time of cancer patients, especially those with brain metastases. Therefore, the appropriate treatment for patients with primary tumor BM requires further study. Meanwhile, the current study has several limitations. This study was a retrospective study, and there is a selection bias in the selection of research objects. Besides, it is difficult to avoid recall bias when obtaining previous information. In addition, as it was a single-center study, there may be selection bias and the sample may not be representative. Moreover, our sample size was not very large, which may limit our power to detect significant associations in multivariate models. Furthermore, unmeasured confounders should not be ruled out, especially the lifestyle factors, which may affect the patients' survival time.

\section{Conclusion}

We analyzed the survival data of patients with BM from the primary tumor. We found that women with a prolonged TTB after primary tumor resection and single intracranial metastasis have prolonged OS. Nevertheless, further epidemiological studies with larger sample sizes are needed to analyze unmeasured confounding factors, especially lifestyle factors, and subsequent multicentres to assess the relationship between primary tumor resection and survival of BM patients.

\section{Funding}

This research was supported by the Research Projects of Public Health Research Center of Jiangnan University in 2018 (Grant No. 1286066903190030).

\section{Conflict of interest}

None to report.

\section{References}

[1] Valiente M, Ahluwalia MS, Boire A, Brastianos PK, Goldberg SB, Lee EQ, et al. The evolving landscape of brain metastasis. Trends Cancer. 2018; 4(3): 176-196. doi: 10.1016/j.trecan.2018.01.003.

[2] Fecci PE, Champion CD, Hoj J, McKernan CM, Goodwin CR, Kirkpatrick JP, et al. The evolving modern management of brain metastasis. Clin Cancer Res. 2019; 25(22): 6570-6580. doi: 10.1158/1078-0432.CCR-18-1624.

[3] Nayak L, Lee EQ, Wen PY. Epidemiology of brain metastases. Curr Oncol Rep. 2012; 14(1): 48-54. doi: 10.1007/s11912011-0203-y.

[4] Soffietti R, Ahluwalia M, Lin N, Rudà R. Management of brain metastases according to molecular subtypes. Nat Rev Neurol. 2020; 16(10): 557-574. doi: 10.1038/s41582-020-0391-x.

[5] Achrol AS, Rennert RC, Anders C, Soffietti R, Ahluwalia MS, Nayak L, et al. Brain metastases. Nat Rev Dis Primers. 2019; 5(1): 5. doi: 10.1038/s41572-018-0055-y. 
[6] Sacks P, Rahman M. Epidemiology of brain metastases. Neurosurg Clin N Am. 2020; 31(4): 481-488. doi: 10.1016/j. nec.2020.06.001.

[7] Siegel RL, Miller KD, Jemal A. Cancer statistics, 2017. CA Cancer J Clin. 2017; 67(1): 7-30. doi: 10.3322/caac.21387.

[8] Imaizumi J, Shida D, Narita Y, Miyakita Y, Tanabe T, Takashima A, et al. Prognostic factors of brain metastases from colorectal cancer. BMC Cancer. 2019; 19(1): 755. doi: 10.1186/s12885-019-5973-x.

[9] Sandler KA, Shaverdian N, Cook RR, Kishan AU, King CR, Yang I, et al. Treatment trends for patients with brain metastases: Does practice reflect the data? Cancer. 2017; 123(12): 2274-2282. doi: 10.1002/cncr.30607.

[10] Leone JP, Lee AV, Brufsky AM. Prognostic factors and survival of patients with brain metastasis from breast cancer who underwent craniotomy. Cancer Med. 2015; 4(7): 989-994. doi: 10.1002/cam4.439.

[11] Pan F, Cui S, Wang W, Gu A, Jiang L. Survival analysis for lung adenosquamous carcinoma patients with brain metastasis. J Cancer. 2018; 9(20): 3707-3712. doi: 10.7150/jca.27441.

[12] Hatiboglu MA, Akdur K, Sawaya R. Neurosurgical management of patients with brain metastasis. Neurosurg Rev. 2020; 43(2): 483-495. doi: 10.1007/s10143-018-1013-6.

[13] Ewend MG, Brem S, Gilbert M, Goodkin R, Penar PL, Varia M, et al. Treatment of single brain metastasis with resection, intracavity carmustine polymer wafers, and radiation therapy is safe and provides excellent local control. Clin Cancer Res. 2007; 13(12): 3637-3641. doi: 10.1158/1078-0432.CCR-06-2095.

[14] Patchell RA, Tibbs PA, Walsh JW, Dempsey RJ, Maruyama Y, Kryscio RJ, et al. A randomized trial of surgery in the treatment of single metastases to the brain. N Engl J Med. 1990; 322(8): 494-500. doi: 10.1056/NEJM199002223220802.

[15] Paek SH, Audu PB, Sperling MR, Cho J, Andrews DW. Reevaluation of surgery for the treatment of brain metastases: Review of 208 patients with single or multiple brain metastases treated at one institution with modern neurosurgical techniques. Neurosurgery. 2005; 56(5): 1021-1034.

[16] Moravan MJ, Fecci PE, Anders CK, Clarke JM, Salama AKS, Adamson JD, et al. Current multidisciplinary management of brain metastases. Cancer. 2020; 126(7): 1390-1406. doi: 10.1002/cncr.32714.

[17] Andrews DW, Scott CB, Sperduto PW, Flanders AE, Gaspar LE, Schell MC, et al. Whole brain radiation therapy with or without stereotactic radiosurgery boost for patients with one to three brain metastases: Phase III results of the RTOG 9508 randomised trial. Lancet. 2004; 363(9422): 1665-1672. doi: 10.1016/S0140-6736(04)16250-8.

[18] Karlsson B, Hanssens P, Wolff R, Söderman M, Lindquist C, Beute G. Thirty years' experience with Gamma Knife surgery for metastases to the brain. J Neurosurg. 2009; 111(3): 449-457. doi: 10.3171/2008.10.JNS08214.

[19] Yamamoto M, Serizawa T, Shuto T, Akabane A, Higuchi Y, Kawagishi J, et al. Stereotactic radiosurgery for patients with multiple brain metastases (JLGK0901): A multi-institutional prospective observational study. Lancet Oncol. 2014; 15(4): 387-395. doi: 10.1016/S1470-2045(14)70061-0.

[20] Lu F, Hou Y, Xia Y, Li L, Wang L, Cao K, et al. Survival and intracranial control outcomes of whole-brain radiotherapy (WBRT) alone versus WBRT plus a radiotherapy boost in non-small-cell lung cancer with brain metastases: A singleinstitution retrospective analysis. Cancer Manag Res. 2019; 11: 4255-4272. doi: 10.2147/CMAR.S203461.

[21] Rades D, Hansen HC, Schild SE, Janssen S. A New Diagnosis-Specific Survival Score for Patients to be Irradiated for Brain Metastases from Non-small Cell Lung Cancer. Lung. 2019; 197(3): 321-6. doi: 10.1007/s00408-019-00223-6.

[22] Langer CJ, Mehta MP. Current management of brain metastases, with a focus on systemic options. J Clin Oncol. 2005; 23(25): 6207-6219. doi: 10.1200/JCO.2005.03.145.

[23] Ellingson BM, Chung C, Pope WB, Boxerman JL, Kaufmann TJ. Pseudoprogression, radionecrosis, inflammation or true tumor progression? challenges associated with glioblastoma response assessment in an evolving therapeutic landscape. $\mathbf{J}$ Neurooncol. 2017; 134(3): 495-504. doi: 10.1007/s11060-017-2375-2.

[24] Eigentler TK, Figl A, Krex D, Mohr P, Mauch C, Rass K, et al. Dermatologic Cooperative Oncology Group and the National Interdisciplinary Working Group on Melanoma. Number of metastases, serum lactate dehydrogenase level, and type of treatment are prognostic factors in patients with brain metastases of malignant melanoma. Cancer. 2011; 117(8): 1697-703. doi: 10.1002/cncr.25631. 